\section{PEDIATRIC SEDATION OUTSIDE OF THE OPERATING ROOM}

Kiera P. Mason, editor

Springer, 2011

In 1889, Ralph Waldo Emerson was quoted as saying: "If a man can write a better book, preach a better sermon, or make a better mousetrap than his neighbor, the world will beat a path to his door." Dr Mason, by engaging a multispecialty international collaboration of medical/dental specialists, has compiled that better book, and clinicians who sedate children outside of the operating room will soon beat a path to her door. There are few reference texts that cover pediatric sedation, and this book, to my knowledge, is the first text totally dedicated to the sedation of children.

In 519 pages with 73 illustrations, the foundations of pediatric sedation and its applications across medical and dental specialties are detailed in a readable, stepwise format. For such a multiauthored text, the structure, flow, and consistency are a rare treat. Kudos to an editor who cannot only draw upon international expertise, but make it all come together in an organized cohesive format.

The fundamental chapters that provide in-depth reviews of the basis of treating the sedated child are excellent and include physiology, the pharmacokinetic and pharmacodynamics aspects of pediatric pharmacology, sedation scales and discharge criteria, physiologic monitoring, and, most importantly, patient assessment. They form the scientific framework for the clinical application of sedation for the pediatric patient.

Following these important introductory chapters, we are taken on an international odyssey of pediatric sedation journeying across the world and across pediatric specialties that are involved in sedation of children, from pediatric gastroenterologists, oncologists, radiology, and emergency medicine to pediatric dentists. The dental section concentrates on the oral routes of sedation as advocated by the American Academy of Pediatric Dentistry and includes, as does all of the other specialty sections, case studies that present challenging clinical scenarios. I found it very interesting to compare and contrast the various specialty sedation guidelines in relation to those in dentistry. In other chapters, the advanced practitioner will also find the latest drugs and techniques such as the use of intravenous dexmedetomidine for procedural sedation.

The running theme throughout this book is patient safety and how we can best sedate children in various environments, some primitive and some highly technical, to provide safe and effective sedation for children. The scope of the book covers the subject totally, from the challenges, limitations, and development of drugs targeted at children to issues relating to billing and the use of complementary and alternative medicine.

I highly recommend this book to any health professional who is involved in the sedation of children outside of the operating room. It is an interesting read and a wonderful reference text.

Mort Rosenberg

Tufts University Schools of Medicine and Dental Medicine Boston, Massachusetts 